\title{
Original article \\ Dosing fentanyl buccal tablet for breakthrough cancer pain: dose titration versus proportional doses
}

\section{Sebastiano Mercadante}

Anesthesia and Intensive Care Unit; Pain Relief and Palliative Care Unit, La Maddalena Cancer Center, Palermo; and Palliative Medicine, University of Palermo, Palermo, Italy

\section{Antonio Gatti}

Chair of Anesthesiology, Tor Vergata University, Rome, Italy

\section{Giampiero Porzio}

Department of Oncology, University of L'Aquila, and Home Care Program "L'Aquila per la vita", L'Aquila, Italy

\section{Claudio Lo Presti}

Pain Therapy, San Filippo Neri Hospital, Rome, Italy

\section{Federica Aielli}

Department of Oncology, University of L'Aquila, and Home Care Program "L'Aquila per la vita", L'Aquila Italy

\section{Claudio Adile}

Anesthesia and Intensive Care Unit; Pain Relief and Palliative Care Unit, La Maddalena Cancer Center, Palermo, Italy

\section{Alessandra Casuccio}

Department of Experimental Biomedicine and Clinical Neuroscience, University of Palermo, Palermo, Italy

\section{Address for correspondence:}

Prof. Sebastiano Mercadante, Anesthesia and Intensive Care Unit \& Pain Relief and Palliative Care Unit, La Maddalena Cancer Center, Via san Lorenzo 312, 90145 Palermo, Italy. Tel: + 390916806521 ; Fax: +39 0916806110;

terapiadeldolore@lamaddalenanet.it,

03sebelle@gmail.com

Key words:

Breakthrough pain - Cancer pain - Dose titration Fentanyl buccal tablet - Rapid onset opioids

Accepted: 3 April 2012; published online: 21 May 2012 Citation: Curr Med Res Opin 2012; 28:963-68

\section{Abstract}

\section{Objectives:}

The aim of this study was to compare the efficacy and safety of doses of fentanyl buccal tablet (FBT) proportional to doses of opioids used for background analgesia versus dose titration starting with the minimal dose for the management of breakthrough cancer pain (BTCP)

\section{Methods:}

A total of 82 cancer patients with $\mathrm{BTCP}$ who were receiving strong opioids in doses of at least $60 \mathrm{mg}$ of oral morphine equivalents and having acceptable background analgesia, were selected for a multicenter unblinded study. Forty-one patients were randomized to receive FBT in doses proportional to the daily opioid doses for four consecutive episodes of BTcP (group P). Forty-one patients underwent dose titration of FBT, with an initial dose of $100 \mu \mathrm{g}$, for four consecutive episodes (group T). Pain intensity and symptoms associated with opioid therapy were measured before administering any dose of FBT (TO) and 15 minutes after (T15).

Results:

In all, 80 patients were considered for analysis (39 and 41 patients in group $\mathrm{P}$ and $\mathrm{T}$, respectively). Patients were receiving a mean of $126 \pm 100 \mathrm{mg}$ of oral morphine equivalents (range $60-480 \mathrm{mg}$ ) for background analgesia. A total of 293 episodes of BTCP (144 and 149 in group P and T, respectively) were treated and considered for analysis. No differences were found in the decrease of pain intensity between the two groups. However, in patients receiving doses of oral morphine equivalents of $>120 \mathrm{mg} /$ day, a significant number of patients obtained a decrease in pain intensity $>50 \%$ in group $\mathrm{P}$ in comparison with group $\mathrm{T}(p=0.040)$. Also, the need for rescue medication was significantly more frequently reported in group $T$ for the first episode of $\mathrm{BTCP}(p<0.0005)$. No differences in the level of adverse effects were observed between the two groups. No differences in patients' satisfaction were reported.

\section{Conclusion:}

According to the data obtained in this study, there is no evidence for the use of dose titration in the management of BTCP in opioid-tolerant patients. Indeed, doses proportional to basal opioid regimen for background pain seem to be effective and safe in the majority of patients. Further studies should confirm this data in patients receiving higher doses of opioids, with other rapid-onset opioids, and in other settings.

\section{Introduction}

Breakthrough cancer pain $(\mathrm{BT} \mathrm{cP})$ has been recently defined as a transitory increase in pain intensity that occurs either spontaneously, or in relation to a specific predictable or unpredictable trigger, despite relatively stable and adequately controlled background pain ${ }^{1}$. 
$\mathrm{BTcP}$ is a common problem in patients with cancer and is associated with significant morbidity ${ }^{2,3}$. In different surveys, $50-90 \%$ of cancer patients with pain have been reported to experience intermittent flares of their pain, although different definitions and methodologies were used $^{1-6}$.

The availability of supplemental doses of oral opioids, in addition to the continuous analgesic medication, is the main suggested treatment for the management of pain flares. Given the temporal pattern of BTcP, characterized by rapid onset and short duration, various technologies have been developed to provide rapid onset of effect with potent opioid drugs such fentanyl (rapid onset opioids, ROOs), delivered by non-invasive routes. It has been suggested that the therapy dose should be individually titrated in order to adapt management of this condition and enable effective analgesia to be delivered while minimizing the risk of clinically significant adverse effects ${ }^{1,7}$. This statement has been quoted as evidence "B". However, the need for titrating opioid doses in $\mathrm{BTcP}$ may make the practical use of ROOs difficult in daily activity, particularly at home or in outpatient clinics. Most patients may be reluctant to try the medication and avoid the use of an $\mathrm{ROO}$, preferring, in the end, traditional oral dosing of morphine ${ }^{8}$. Moreover, the need for dose titration with ROOs in BTcP, has never been properly assessed and this statement is derived from a series of papers published for regulatory issues. To scientifically affirm the need for titration, a randomized trial should compare a group of patients titrated versus another group of patients who receive proportional doses, and this study design has never been the subject of research. For instance, there is no evidence for dose titration as well as for using proportional doses ${ }^{9,10}$. The aim of this randomized-controlled study was to compare efficacy and safety of doses of an ROO (fentanyl buccal tablet, FBT), given in doses proportional to opioid daily doses with dose titration, starting with the lowest dose of FBT for the management of $\mathrm{BTcP}$. The primary outcomes were the number of episodes requiring rescue medication after the study dose and the number of episodes with a decrease in pain intensity of $\geq 33 \%$ and $\geq 50 \% 15$ minutes after the study dose. The secondary outcome was the number of episodes in which patients reported adverse effects and the level of satisfaction with the treatments.

\section{Patients and methods}

A multicenter prospective randomized controlled nonblinded study was carried out in a sample of 82 cancer patients with $\mathrm{BTcP}$ who were receiving strong opioids in doses of at least $60 \mathrm{mg}$ of oral morphine equivalents and had an acceptable background analgesia ( $\leq 4$ on a numerical scale from 0 to 10 ). Patients with more than three episodes of $\mathrm{BTcP} /$ day, relevant co-existing liver or renal disease, cognitive impairment, an expected survival less than 3 months, requiring radiotherapy, or a new course of chemotherapy, were excluded. The study was approved by the local ethical committee of the University of Palermo, and adhered to Helsinki Declaration (Eudract number 03125514, 2010). Informed consent was taken from all patients before participation.

Randomization was computer-generated at the principal investigation center. A total of 41patients were randomized to receive FBT in doses proportional to the daily opioid doses (for example $100 \mu \mathrm{g}$ for $60 \mathrm{mg} /$ day of oral morphine, $200 \mu \mathrm{g}$ for $120 \mathrm{mg} /$ day or oral morphine, and so on) for four consecutive episodes of BTcP (group P). A total of 41 patients underwent dose titration of FBT, with an initial dose of $100 \mu \mathrm{g}$. In this group, when $\mathrm{BTcP}$ was considered to be unsuccessfully controlled, the dose was progressively increased for the subsequent episodes, up to 200, 400 and $800 \mu \mathrm{g}$, to achieve the effective dose (group $\mathrm{T})$. For each episode it was considered the percentage of decrease in pain intensity ( $\geq 33 \%$ and $50 \%$, respectively), 15 minutes after administration of FBT (see below).

Age, gender, primary cancer, pain causes and mechanisms on the basis of clinical history, known metastases, physical examination, and available investigations were recorded (particularly pain on movement due to bone metastases). The following parameters were collected: pain intensity, measured on a numerical rating scale from 0 to $10^{11}$ before administering any dose of FBT (T0) and 15 minutes after (T15), symptoms associated with opioid therapy, such as nausea and vomiting, drowsiness, and confusion, occurring as a new event or a change in intensity as a consequence of FBT administration, assessed by patients, by using a scale from 0 to 3 (not at all, slight, a lot, severe). At the end of the study, patients were asked about their satisfaction regarding the treatment, rated on a verbal scale: excellent, good, sufficient, poor, very poor. Efficacy was assessed by differences in pain intensity for each episode, by the number of episodes successfully treated (decrease of $\geq 33$ and $\geq 50 \%$ in pain intensity), by the use of rescue doses, by the level of patient's satisfaction, and the number of patients who discontinued the treatment for poor compliance or inefficacy. Safety was assessed by the differences in opioids-related adverse effects or number of patients who discontinued the treatment for adverse effects.

\section{Statistical analysis}

Statistical analysis of quantitative and qualitative data, included descriptive statistics, was performed for all the items. Frequency analysis was performed by chi-square test with Yates correction. The paired samples Student's $t$-test was used to compare mean pain intensity scores in 
the time periods. The two-sample Student's t-test was used to compare parametric variables between groups. All $p$-values were two-sided and $p$-values less than 0.05 were considered to indicate statistical significance. Data were analyzed by the Epi Info software, version 3.2.2, (Centers for Disease Control and Prevention, USA) and the SPSS Software 14.0 version (SPSS, Inc.,Chicago, IL, USA).

\section{Results}

Patients characteristics are described in Table 1. Two patients in group $\mathrm{P}$ were receiving doses of less than $60 \mathrm{mg}$ of oral morphine equivalents and were excluded from the study. In all, 80 patients were considered for analysis (39 and 41 patients in group $\mathrm{P}$ and $\mathrm{T}$, respectively). Patients were receiving a mean of $126 \pm 100 \mathrm{mg}$ of oral morphine equivalents (range $60-480 \mathrm{mg}$ ) for background analgesia.

A total of 293 episodes of BTcP (144 and 149 in group P and $\mathrm{T}$, respectively) were treated and considered for analysis. In all, 71 patients ( 36 and 35 in group $\mathrm{P}$ and $\mathrm{T}$, respectively) received the selected doses for all the four episodes of BTcP. Five patients were excluded because they did not present episodes of $\mathrm{BTcP}$ within 3 days (three and two patients in group $\mathrm{P}$ and $\mathrm{T}$, respectively). One of the patients in group $\mathrm{T}$ was transferred to another unit. Four patients in group $\mathrm{T}$ discontinued the treatment due to poor compliance or inefficacy of the treatment and not all the four episodes were recorded (see Figure 1).

Changes in pain intensity, number of episodes with a decrease in pain intensity $\geq 33 \%$ and $\geq 50 \%, 15$ minutes after the administration of the selected dos of FBT in group $\mathrm{P}$ and $\mathrm{T}$ are shown in Table 2 . The same data in 30 patients who were receiving oral morphine equivalents of $\geq 120 \mathrm{mg} /$ day are presented in Table 3. A significant number of patients obtained a decrease in pain intensity $\geq 50 \%$ in group $\mathrm{P}$ in comparison with group $\mathrm{T}$ patients $(p=0.040)$. The need for rescue medication was significantly more frequently reported in group $\mathrm{T}$ for the first episode of BTcP. Eight and 25 episodes in group P and

Table 1. Characteristics of patients.

\begin{tabular}{lccc}
\hline & Group P & Group T & $p$-value \\
\hline Age (mean \pm SD, years) & $61.3(9.7)$ & $65.4(10.5)$ & $0.355^{*}$ \\
Gender (M/F) & $21 / 15$ & $17 / 18$ & $0.410 \dagger$ \\
Primary tumor & & & \\
$\quad$ Lung & 6 & 6 & \\
$\quad$ Gastrointestinal & 5 & 10 & \\
$\quad$ Breast & 3 & 3 & \\
$\quad$ Urogenital & 8 & 7 & \\
$\quad$ Other & 14 & 9 & $0.173 \dagger$ \\
Incident pain due to & $19 / 36$ & $24 / 35$ & \\
$\quad$ bone metastases & & & \\
\hline
\end{tabular}

*Two-sample $t$-test; †Pearson chi-square statistic test with Yates correction.
$\mathrm{T}$, respectively, required a rescue dose after the selected dose of FBT $(p<0.0005)$. This difference was less relevant for the subsequent episodes: rescue doses were given in seven and 11 episodes, respectively, for the second episode of BTcP $(p=0.341)$; in six and four episodes respectively, for the third episode of BTcP $(p=0.437)$; and in five and one episodes respectively, for the fourth episode of BTcP $(p=0.088)$.

No patient developed adverse effects of severe intensity in both groups. The number of patients who developed adverse effects of mild-to-moderate intensity, attributable to study medication are reported in Tables 2 and 3. No differences in adverse effects intensity was observed between the two groups. No differences in patients' satisfaction were reported ( $p=0.106$, Pearson chi-square statistic test).

\section{Discussion}

Data from this study suggest that dose titration does not add any advantage in comparison with proportional doses in the management of $\mathrm{BTcP}$, being neither more efficacious nor safer. Doses proportional to basal opioid regimen for background pain are effective, providing optimal analgesia in the majority of treated episodes of $\mathrm{BTcP}$, and are devoid of important adverse effects. Indeed, more patients who underwent dose titration discontinued the treatment for poor efficacy or compliance and required more rescue medications after receiving FBT. As expected, patients given similar doses of FBT of $100 \mu \mathrm{g}$ in both arms reported similar outcomes, because they were receiving $60 \mathrm{mg}$ of oral morphine equivalents for their background analgesia. Indeed, differences were observed in patients receiving higher doses of oral morphine equivalents $(\geq 120 \mathrm{mg} /$ day), when proportional doses were higher than the initial titration dose. This better outcome was obtained without more adverse effects being reported. This can be explained by the tolerance relative to opioid dose regimen. Finally, patients' satisfaction did not differ between the two groups.

The choice of the dose of an ROO to be prescribed for $\mathrm{BT} \mathrm{CP}_{\mathrm{C}}$ has been controversial. Based on analysis of the literature, recent recommendations have suggested that the dose of ROOs to be given for an episode of $\mathrm{BTcP}$ should be determined by individual titration ${ }^{1}$. However, there are several considerations regarding this statement that should be taken into account. First, about $10-30 \%$ of episodes treated may fail during dose titration, especially in patients receiving high doses of opioids ${ }^{9,10}$. Secondly, dose titration may make the practical use of ROOs difficult in the daily activity, particularly at home or in outpatient clinics. Considering how presentation differs for each episode, titration should potentially be performed in each case. Thirdly, using different pieces of ROO for treating each episode may be time consuming and may well exceed 
82 patients

Group P

2 patients receiving less than $60 \mathrm{mg}$

of oral morphine equivalents
Group T

80 patients

Group P

39 patients

36 patients: 4 episodes treated

3 patients: absence of episodes

\section{Group T}

41 patients

35 patients: 4 episodes treated 2 patients: absence of episodes 4 patients discontinued due to poor efficacy or compliance: -2 patients: 3 episodes treated - 1 patient: 2 episodes treated -1 patient: 1 episode treated

Number of BTcP episodes treated and considered for comparison

Group P

144
Group T

149

Figure 1. CONSORT flow chart of the study.

Table 2. Pain intensity differences between the two groups for all the episodes of BTcP $(n=284)$.

\begin{tabular}{lccc}
\hline & Group P & Group T & $p$-value \\
\hline T0, mean $( \pm S D)$ & $7.6(1.0)$ & $8.1(1.2)$ & \\
T15, mean $( \pm S D)$ & $3.1(1.7)^{*}$ & $3.1(1.4)^{*}$ & \\
No. of BTcP episodes & & & \\
$\quad$ With a decrease $>33 \%$ & $123 / 144$ & $119 / 140$ & $0.921 \dagger$ \\
$\quad$ With a decrease $>50 \%$ & $112 / 144$ & $104 / 140$ & $0.490 \dagger$ \\
No. of BTcP episodes & \\
$\quad$ With AE intensity 1-2 & $40 / 144$ & $47 / 140$ & $0.289 \dagger$ \\
\hline
\end{tabular}

$* p<0.0005$ vs. T0 (paired samples $t$-test); $\uparrow$ Pearson chi-square statistic test with Yates correction; ${ }^{*}$ Two-sample $t$-test.

the duration of $\mathrm{BTcP}$ which can subside spontaneously, as evidenced by successful placebo-treated patients reported in literature 9 . From a practical point of view, patients should be prescribed more packages with different doses, unless using more pieces of lower doses. Most patients may
Table 3. Pain intensity differences between the two groups for episodes of $\mathrm{BTcP}$ in patients receiving doses of oral morphine equivalents $\geq 120 \mathrm{mg} /$ day ( 60 and 60 episodes in groups $P$ and $T$, respectively).

\begin{tabular}{lccc}
\hline & Group P & Group T & $p$-value \\
\hline T0, mean $( \pm S D)$ & $7.3(0.9)$ & $8.4(0.9)$ & \\
T15, mean $( \pm S D)$ & $2.8(1.6)^{*}$ & $3.7(1.3)^{*}$ & \\
No. of BTcP episodes & & & \\
$\quad$ With a decrease in PI $>33 \%$ & $52 / 60$ & $45 / 60$ & $0.104 \dagger$ \\
$\quad$ With a decrease in Pl $>50 \%$ & $49 / 60$ & $38 / 60$ & $0.040 \dagger$ \\
No. of BTcP episodes & & & \\
$\quad$ With AE intensity 1-2 & $22 / 60$ & $21 / 60$ & $1.0 \dagger$ \\
\hline
\end{tabular}

$* P<0.0005$ vs. T0 (paired samples $t$-test); $\uparrow$ Pearson chi-square statistic test with Yates correction; ${ }^{*}$ Two-sample $t$-test.

be reluctant to try the ROO medication and avoid using these drugs, preferring, in the end, traditional oral dosing of morphine $e^{4}$. Fourthly, randomized trials that state the need of dose titration, have never specifically examined this issue, and the information gathered is only a 
consequence of the study design which was aimed to demonstrate the superiority of ROOs over placebo, oral morphine or usual oral opioids, or to evaluate the safety and efficacy of ascending doses of ROOs in dose-finding studies. The titration period was open and not confirmed by comparison with any other method. The reason for the lack of relationship between doses for BTcP and a basal opioid regimen have not been clearly explained, considering that the presence of tolerance would suggest a dose proportional to that used for background analgesia. Of interest, tolerance to adverse effects in patients chronically exposed to opioids has been found, despite serum fentanyl levels as high as $6-8 \mathrm{ng} / \mathrm{mL}^{12}$.

Finally, despite a large interindividual variability in patients' dose requirements, observations from data pooled from the same oral transmucosal fentanyl citrate (OTFC) trials showed a statistically significant relationship between the BTcP and round-the-clock opioid dose, also considering that the protocol was not aimed at demonstrating this ${ }^{13}$. When data were available, a simulation of a calculation of doses of opioids used for background analgesia and those achieved after individual titration showed mean values of proportional doses very close to those found after titration ${ }^{14}$.

It could be argued that a proportional dose without titration could expose patients to adverse effects. In practice, although dose titration may appear safer, it requires doses similar to those resulting from proportional doses and may results in more failures, prolonging patients' suffering and reducing their compliance. In a previous small controlled study comparing the use of OTFC and intravenous morphine given at doses proportional to the daily opioid dosage ${ }^{15}$, both intravenous morphine and OTFC were effective without producing relevant adverse effects, even when started at high doses in highly tolerant patients. In a study reproducing a clinical scenario of patients receiving opioids for $\mathrm{BTcP}$, the dose of oral opioids used as rescue medication was $18 \%$ of the round-the-clock opioid dose, whereas for OTFC, titrated to determine the effective dose, the rescue dose was about $35 \%$ of the roundthe-clock dose ${ }^{16}$, suggesting that the titration process may provide even higher doses than those expected by using proportional doses to the basal regimen.

Preliminary and confirmatory surveys have shown the safety of this approach in a large number of patients with no life-threatening adverse effects which occurs even in older patients treated with large doses of intravenous morphine. Respiratory depression, which is the most feared adverse effect, has never occurred, and no emergency call was needed ${ }^{17-19}$. As intravenous morphine has the highest intrinsic risk for serious adverse events, one could argue that other drugs with similar rapid effects should be at least as safe. On the other hand experience is quite limited in patients receiving high doses of opioids. All the studies were based on limited maximum doses of the different ROOs (OTFC, FBT, intranasal fentanyl and sublingual fentanyl, $1600,800,200$, and $800 \mu \mathrm{g}$, respectively. For instance, in a recent preliminary study of the use of FBT in patients receiving high doses of opioids, proportionally higher doses of FBT were effective and well-tolerated by most patients ${ }^{20}$. This can be explained by the protective effect offered by opioid tolerance in patients chronically receiving relevant opioid doses for the management of cancer pain.

Among the ROOs available at the time of planning the current study, FBT was chosen because of its availability, greater independence from variability associated with the individual use, for example that found with OTFC. The interval of 15 minutes was chosen to evaluate the effectiveness of the treatment, as FBT is expected to produce analgesia within this period ${ }^{21}$.

The principal limitation of this study is the lack of blindness. Regardless of this, the complexity of this procedure in this context, and the associated costs for a study which was spontaneous and not sponsored must be borne in mind. In the same way as the current study design, all titration phases reported in the literature were open and not controlled, as were other studies assessing the efficacy and the safety of opioids given in doses proportional to the opioid basal regimen ${ }^{17-19}$. These experiences have shown that in patients receiving opioids for chronic cancer pain the risks of administering about $20 \%$ of the daily dose of opioids with rapid modalities, intravenously or transmucosally, are minimal, going back to previous recommendations of European Association for Palliative Care, based on clinical experience ${ }^{22}$. The sample of patients was typical in terms of opioid treatment for background analgesia, ranging from 60 to $480 \mathrm{mg}$ of oral morphine equivalents.

It is likely that differences could be more relevant in a selected population of patients receiving high doses of opioids, as suggested by data of patients receiving $\geq 120 \mathrm{mg} /$ daily of oral morphine equivalents.

In conclusion, a titration process starting with the lowest doses of FBT, does not add any advantage in comparison with proportional doses in the management of $\mathrm{BTcP}$, being neither more efficacious or safer. Doses proportional to basal opioid regimen for background pain seem to be effective and safe in the majority of patients. From a practical perspective, to reduce patients' reluctance in asking for opioid rescue medication $8,16,23$, a definite indication should be provided and self-titration is unlikely to be accepted, unless performed in an assisted setting. Practical problems, including the need of titration, has probably limited the use of ROOs, despite the superiority over oral opioids and placebo demonstrated in various studies, regardless of the cost. As a consequence many patients will continue to prefer the conventional use of oral morphine, even though most of the episodes will evanish spontaneously ${ }^{8}$, and an uneventful burst of morphine will be gratuitously given. It is also to underline that all the 
pioneer controlled studies were designed for approval from FDA. Therefore, indications were dictated by the prudence of managing new delivery systems, which were in their infancy. Further studies should be performed to confirm the data with other delivery systems, selected population, such as elderly, or patients receiving high doses of opioids for chronic cancer pain.

\section{Transparency}

\section{Declaration of funding}

The authors received no payment in preparation of this manuscript.

\section{Declaration of financial/other relationships}

The authors declare no conflict of interest.

$\mathrm{CMRO}$ peer reviewers may have received honoraria for their review work. The peer reviewers on this manuscript have disclosed that they have no relevant financial relationships.

\section{References}

1. Davies AN, Dickman A, Reid C, et al. The management of cancer-related breakthrough pain: recommendations of a task group of the Science Committee of the Association for Palliative Medicine of Great Britain and Ireland. Eur J Pain 2009;13:331-8

2. Portenoy RK, Hagen NA. Breakthrough pain: definition, prevalence and characteristics. Pain 1990:41:273-81

3. Portenoy RK, Payne D, Jacobson P. Breakthrough pain: characteristics and impact in patients with cancer pain. Pain 1999;81:129-34

4. Caraceni A, Martini C, Zecca E, et al. Working Group of an IASP Task Force on Cancer Pain. Breakthrough pain characteristics and syndromes in patients with cancer pain. An international survey. Palliat Med 2004:18:177-83

5. Haugen DF, Hjermstad MJ, Hagen N, et al. Assessment and classification of cancer breakthrough pain: a systematic literature review. Pain 2010;149:47682

6. Mercadante S, Radbruch L, Caraceni A, et al. Episodic (breakthrough) pain. Cancer 2002:94:832-9

7. Kleeberg U, Filbet M, Zeppetella G. Fentanyl buccal tablet for breakthrough cancer pain: why titrate? Pain Pract 2011;11:185-90
8. Davies A, Vriens J, Kennett A, et al. An observational study of oncology patients' utilization of breakthrough pain medication. J Pain Symptom Manage 2008;35:406-11

9. Mercadante S. Breakthrough pain: on the road again. Eur $J$ Pain 2009:13:329-30

10. Mercadante $\mathrm{S}$. The use of rapid onset opioids for breakthrough cancer pain: The challenge of its dosing. Crit Rev Oncol Hematol, 2011;80:460-5

11. Hjermstad MJ, Fayers PM, Haugen DF, et al. European Palliative Care Research Collaborative (EPCRC). Studies comparing numerical rating scales, verbal rating scales, and visual analogue scales for assessment of pain intensity in adults: a systematic literature review. J Pain Symptom Manage 2011;41:1073-93

12. Aronoff $G$, Brennan $M$, Pritchard D, et al. Evidence-based oral transmucosal fentanyl citrate (OTFC) dosing guidelines. Pain Med 2005;6:305-14

13. Hagen NA, Fisher $K$, Victorino $C$, et al. A titration strategy is needed to manage breakthrough cancer pain effectively: observations from data pooled from three clinical trials. J Palliat Med 2007:10:47-55

14. Mercadante S. Rapid onset opioids for breakthrough pain: titrating or not titration or not titrating, this is the question. Eur J Pain 2011(Suppl):443-8

15. Mercadante $S$, Villari $P$, Ferrera $P$, et al. Transmucosal fentanyl vs intravenous morphine in doses proportional to basal opioid regimen for episodic-breakthrough pain. Br J Cancer 2007;96:1828-33

16. Zeppetella G. Opioids for cancer breakthrough pain: a pilot study reporting patient assessment of time to meaningful pain relief. J Pain Symptom Manage 2008:35:563-7

17. Mercadante $\mathrm{S}$, Intravaia $\mathrm{G}$, Villari $\mathrm{P}$, et al. Intravenous morphine for episodicbreakthrough pain in an acute palliative care unit: a confirmatory study. J Pain Symptom Manage 2008;35:307-13

18. Mercadante $\mathrm{S}$, Villari $\mathrm{P}$, Ferrera $\mathrm{P}$, et al. The use of opioids for breakthrough pain in an acute palliative care unit by using doses proportional to opioid basal regimen. Clin J Pain 2010;26:306-9

19. Mercadante S, Villari P, Ferrera P, et al. Safety and effectiveness of intravenous morphine for episodic (breakthrough) pain using a fixed ratio with the oral daily morphine dose. J Pain Symptom Manage 2004;27:352-9

20. Mercadante S, Ferrera P, Adile C, et al. The use of fentanyl buccal tablets in doses proportional to the doses of opioids used for background analgesia for the treatment of breakthrough cancer pain in highly tolerant cancer patients. J Pain Symptom Manage 2011:42:464-9

21. Darwish M, Kirby M, Robertson P, et al. Dose proportionality of fentanyl buccal tablets in doses ranging from 600 to $1300 \mu \mathrm{g}$ in healthy adult subjects. A randomized, open-label, four period, crossover, single-centre study. Clin Drug Investig 2010;30:365-78

22. Hanks GW, De Conno F, Cherny N, et al. Morphine and alternative opioids in cancer pain: the EAPC recommendations. Br J Cancer 2001;84:587-93

23. Walker $G$, Wilcock A, Manderson C, et al. The acceptability of different routes of administration of analgesia for breakthrough pain. Palliat Med 2003:17:219-21 